\title{
Regulated expression of claudin-4 decreases paracellular conductance through a selective decrease in sodium permeability
}

\author{
Christina Van Itallie, ${ }^{1}$ Christoph Rahner, ${ }^{1}$ and James Melvin Anderson ${ }^{1,2}$ \\ ${ }^{1}$ Department of Internal Medicine, and \\ ${ }^{2}$ Department of Cell Biology, Yale University School of Medicine, New Haven, Connecticut, USA \\ Address correspondence to: Christina Van Itallie, Department of Internal Medicine, 1080 LMP, PO Box 208019, \\ Yale University School of Medicine, 333 Cedar St., New Haven, Connecticut 06520-8019, USA. \\ Phone: (203) 785-4133; Fax: (203) 785-7273; E-mail: christina.vanitallie@yale.edu.
}

Received for publication February 7, 2001, and accepted in revised form April 10, 2001.

Tight junctions regulate paracellular conductance and ionic selectivity. These properties vary among epithelia but the molecular basis of this variation remains unknown. To test whether members of the claudin family of tight junction proteins influence paracellular ionic selectivity, we expressed human claudin-4 in cultured MDCK cells using an inducible promoter. Overexpression increased the complexity of tight junction strands visible by freeze-fracture microscopy without affecting the levels of claudin-1, -2 , or -3 , occludin, or ZO-1. A decrease in conductance correlated directly with the kinetics of claudin- 4 induction. Dilution potentials revealed that the decrease in paracellular conductance resulted from a selective decrease in $\mathrm{Na}^{+}$permeability without a significant effect on $\mathrm{Cl}^{-}$permeability. Flux for an uncharged solute, mannitol, and the rank order of permeabilities for the alkali metal cations were unchanged. A paracellular site for these effects was supported by the lack of apical/basal directionality of the dilution potentials, the linearity of current-voltage relationships, and the lack of influence of inhibitors of major transcellular transporters. These results provide, to our knowledge, the first direct demonstration of the ability of a claudin to influence paracellular ion selectivity and support a role for the claudins in creating selective channels through the tight-junction barrier.

J. Clin. Invest. 107:1319-1327 (2001).

\section{Introduction}

The tight junction forms a regulated barrier to paracellular transport of solutes and ions (1-3). The barrier contains aqueous channels capable of discriminating charge and size. Both overall electrical conductance and specific ion selectivity differ widely among epithelia. The molecular basis of these variable physiologic properties remains poorly understood. Recently, a family of transmembrane proteins called claudins was identified and localized to within the tight junction (4, $5)$. Indirect evidence suggests claudins might create the selective paracellular properties $(6,7)$. In the present study we directly test whether overexpression of one claudin, claudin- 4 , can confer ionic discrimination to the paracellular pathway.

The 20 members of the claudin family (8) share several essential features. They are small proteins, $20-24 \mathrm{kDa}$, with cytoplasmic amino and carboxyl termini and two extracellular domains. The carboxyl terminus of most claudins ends in a putative PDZ-binding domain, and interaction of claudins $1-8$ with the first PDZ domains of the tight-junction plaque proteins $\mathrm{ZO}-1,-2$, and -3 has been demonstrated in vitro (9). The extracellular domains (about 57 and 25 amino acids, respectively) share regions of sequence identity, but are quite variable in the position and number of amino acids with charged side chains, suggesting they might have different effects on the paracellular diffusion of ions. Freeze-fracture micrographs reveal the barrier is formed where continuous rows of transmembrane proteins from opposing cells make adhesive contacts in the intercellular space. These strands are composed of claudins $(7,10)$.

Characterization of the claudins in vivo and in cultured cell models is beginning to offer functional insights. Expression of claudin-11/OSP is concentrated in testis and oligodendrocytes of the central nervous system (CNS). Deletion of the claudin-11 gene in mice results in disappearance of tight junction fibrils in testis and male sterility, as well as delayed axonal conduction rates in the CNS (7). Human mutations in claudin16/paracellin implicate this claudin in paracellular magnesium reabsorption in the thick ascending loop of Henle (6). An autosomal recessive form of deafness was associated recently with human mutations in claudin14; the authors speculated that this was related to inability to maintain the special ionic environments in the cochlear duct (11). In Madin-Darby canine kidney (MDCK) cells, an in vitro epithelial model, overexpression of claudin-1 increases transepithelial electrical resistance (TER) with variable effects on mannitol flux $(12,13)$. Consistent with a presumed role in creating the tissue-specific physiologic properties, some claudins have very restricted and different distribution patterns among cell types and tissues (14-16). 
In the present study we expressed human claudin-4 in cultured MDCK cell monolayers under an inducible promoter and characterized changes in junction structure and physiology. We observed that overexpression of claudin-4 decreased paracellular electrical conductance due to a selective decrease in $\mathrm{Na}^{+}$permeability, with no significant change in the permeability for $\mathrm{Cl}^{-}$. This is the first demonstration that a claudin can confer ionic selectivity to paracellular transport and leads to the prediction that the combination of different claudins defines the overall selectivity of different junctions.

\section{Methods}

Plasmid cDNA constructs. Full-length human claudin-4 was amplified by PCR using human kidney cDNA (Quick-clone cDNA; CLONTECH Laboratories Inc., Palo Alto, California, USA) as a template and primer 56948 (5'-CGGGATCCCTGACAATGGCCTCCATGGGGCTAC-3'), primer 56949 (5'-GCTCTAGATTACACGTAGTTGCTGGCAGC-3'), and Taq polymerase (Promega Corp., Madison, Wisconsin, USA). The amplified product was cloned into the TOPO-TA vector (Invitrogen Corp., San Diego, California, USA) and subcloned using EcoRI into the pTRE vector (CLONTECH Laboratories Inc.). DNA sequence was verified in both directions.

Cell culture and generation of stable lines. Human claudin4 was transfected into tet-off MDCK II cells (CLONTECH Laboratories Inc.) along with 20-fold less pTKhyg selection plasmid using the Lipofectamine protocol (Life Technologies Inc., Grand Island, New York, USA). Claudin-4-transfected clones were selected in $0.2 \mathrm{mg} / \mathrm{ml}$ hygromycin $\mathrm{B}$, and resistant clones were maintained in high-glucose DMEM supplemented with $10 \%$ FBS, penicillin/streptomycin, $0.1 \mathrm{mg} / \mathrm{ml}$ hygromycin, and $50 \mathrm{ng} / \mathrm{ml}$ doxycycline. Cell lines were screened by immunoblotting. For experiments, cells were trypsinized and replated onto Snapwells (Corning Inc. Life Sciences, Acton, Massachusetts, USA) at $5 \times$ $10^{4}$ cells/well in doxycycline-containing $(50 \mathrm{ng} / \mathrm{ml})$ or doxycycline-free media (high-glucose DMEM supplemented with penicillin/streptomycin and 10\% FBS, CLONTECH Laboratories Inc.-certified tetracyclinefree serum). Cells plated for experiments were refed daily with the appropriate media.

Immunofluorescence microscopy. Cells were grown on filters (Transwell-Clear; Corning Inc. Life Sciences), and all methods for immunolocalization have been described previously (17). ZO-1 was detected with a mouse mAb (1:300; Zymed Laboratories Inc., South San Francisco, California, USA); claudin-4 was detected using a rabbit polyclonal $\mathrm{Ab}$ to human claudin-4 (1:100; Zymed Laboratories Inc.). Secondary Ab's were Cy3-labeled anti-mouse and Cy-2-labeled affinity-purified anti-rabbit species-specific Ab's (Jackson ImmunoResearch Laboratories Inc., West Grove, Pennsylvania, USA). All microscopy was performed on a Nikon Microphot FX microscope using a ×60 PlanApo lens, and images were captured using a Sensys cooled
CCD camera. Images were then processed using OpenLab software (Improvision Inc., Lexington, Massachusetts, USA) and Adobe Photoshop 5.0 (Adobe Systems Inc., San Jose, California, USA).

Freeze-fracture electron microscopy. Freeze-fracture analysis of uninduced and induced MDCK cells was performed as described previously (18). Replica sets from two separate uninduced and induced MDCK cell clones were examined, and the freeze-fracture patterns were similar for each.

Immunoblot analysis. To determine the expression profiles of claudin-4-overexpressing cell lines, transfected MDCK cells were plated in twelve-well dishes on Trans-well filters. No differences in kinetics or levels of protein induction were observed associated with the plating substrate. Protein expression was induced by doxycycline removal for 4 days; claudin- 4 protein levels were always compared with that seen in uninduced cells of the same clonal line. Doxycycline had no apparent effect on cell number or cell size; endogenous claudin-4 levels were unaffected by doxycycline treatment in the parental cell line (data not shown). Sample preparation was performed as described previously (18). When cells were grown on filters, the entire filter was removed and placed into 100-200 $\mu \mathrm{l}$ of sample buffer. Samples were stored at $-80^{\circ} \mathrm{C}$ before analysis. Equal volumes of lysate were subjected to SDS-PAGE (13\% polyacrylamide gels for claudin, $8 \%$ gels for ZO-1 and occludin) and transferred to nitrocellulose. Ab's (all from Zymed Laboratories Inc.) used for immunoblot analysis were: claudin-1/3 (1:1000; this lot of claudin- $1 \mathrm{Ab}$ cross reacts with claudin- 3 and possibly claudin-20) and claudin-4 polyclonal (1:1000) Ab's, claudin-2 mouse mAb (1:2500), antihuman ZO-1 mouse mAb (1:1000), and anti-human occludin mAb (1:1000). Antigen-Ab complexes were detected with horseradish peroxidase-conjugated (HRP-conjugated) secondary Ab's by enhanced chemiluminescence (ECL) (Amersham Pharmacia Biotech Inc., Piscataway, New Jersey, USA).

Electrophysiologic measurements. Studies were performed on cell monolayers grown on porous filters (Snapwell; Corning Inc. Life Sciences) in modified Ussing chambers (Navicyte; Harvard Apparatus Co., Holliston, Massachusetts, USA) with a microcomputercontrolled voltage/current clamp (Harvard Apparatus Co.). Temperature was maintained at $37^{\circ} \mathrm{C}$. Solution mixing and $\mathrm{pH}$ were regulated by bubbling water-saturated $95 \% \mathrm{O}_{2}-5 \% \mathrm{CO}_{2}$ through the chambers. Voltage and current electrodes were $\mathrm{Ag} / \mathrm{AgCl}$ electrodes with microporous ceramic tips.

Typical experiments consisted of the following procedure. At the start of each experiment, fluid resistance and electrode potentials were determined across blank filters in buffer A $(120 \mathrm{mM} \mathrm{NaCl}, 10 \mathrm{mM}$ HEPES, pH 7.4, $5 \mathrm{mM} \mathrm{KCl}, 10 \mathrm{mM} \mathrm{NaHCO}_{3}, 1.2 \mathrm{mM} \mathrm{CaCl}_{2}, 1 \mathrm{mM}$ $\mathrm{MgSO}_{4}$ ) (19) and subtracted from subsequent measurements. Next, electrical potentials were determined when buffer B (60 mM NaCl, $120 \mathrm{mM}$ mannitol, 10 


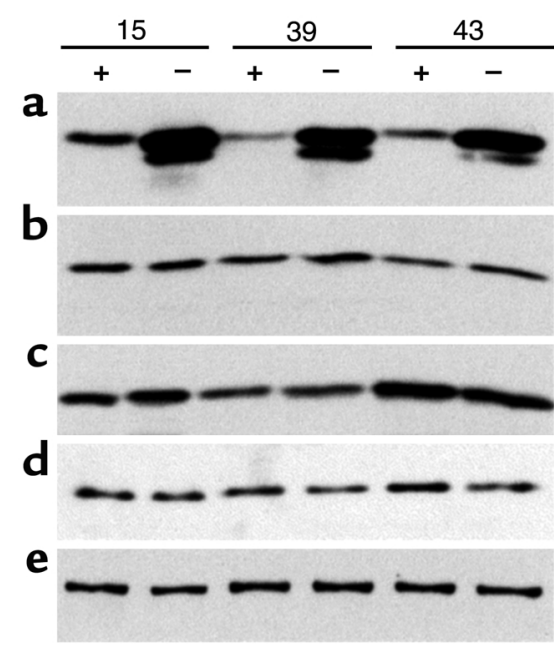

Figure 1

Immunoblot analysis of induction in three separate claudin-4-transfected tet-off MDCK cell clones, 15, 39, and 43. Cells were cultured on filters for 4 days in the presence (+) or absence $(-)$ of $50 \mathrm{ng} / \mathrm{ml}$ doxycycline. Multiple aliquots from the same samples were separated by SDS-PAGE, followed by immunoblotting for (a) claudin-4, (b) claudin$1 / 3$, (c) claudin-2, (d) occludin, and (e) ZO-1. The claudin-4 blot is deliberately overexposed to reveal protein expression in uninduced tetoff cells; the lower band is due to proteolysis during sample preparation. Claudin-4 overexpression has no apparent effect on the other measured tight-junction proteins. Presence or absence of doxycycline has no effect on claudin-4 expression in untransfected MDCK cells.

mM HEPES, pH 7.4, 5 mM KCl, $10 \mathrm{mM} \mathrm{NaHCO}_{3}, 1.2$ $\mathrm{mM} \mathrm{CaCl}_{2}, 1 \mathrm{mM} \mathrm{MgSO}_{4}$ ) replaced buffer $\mathrm{A}$ on the apical or basal side of the blank filters. Dilution potentials measured using blank filters were subtracted from those determined with control or induced MDCK cells.

After initial measurements were made using blank filters, the filters were removed and replaced with those containing confluent MDCK cell monolayers. Filters were immediately washed twice on both the apical and basal surfaces with buffer $A$ at $37^{\circ} \mathrm{C}$. TER or total conductance $\left(G_{T} ; 1 /\right.$ TER $\left.\times 1000\right)$ was measured by the voltage deflection when repetitive $40-\mu \mathrm{A}$ current pulses were passed across the monolayer. Base-line transmonolayer potentials were determined. Stability of conductance and transmonolayer potential measurements were confirmed by repeated measurements (every 6 seconds) for at least 1 minute. Apical buffer was then aspirated and replaced (with one wash) by warmed buffer B. Measurement of conductance and the transmonolayer potential were performed immediately (every 6 seconds) and monitored for at least 1 minute to verify stability. Filters were then removed, and, where indicated, filters were excised and cells extracted for immunoblot analysis. In some cases, the effect of substituting buffer B in the basal compartment was also determined.

Bi-ionic potentials were determined by apical substitution of $120 \mathrm{mM} \mathrm{NaCl}$ with equimolar amounts of $\mathrm{KCl}, \mathrm{LiCl}, \mathrm{RbCl}$, or $\mathrm{CsCl}$. Transmonolayer electrical potential was measured as described above. Measure- ments made using blank filters were subtracted from those determined with cells.

Instantaneous current-voltage relationship was determined in solution A, stepping the voltage from $30 \mathrm{mV}$ to $-30 \mathrm{mV}$ in steps of $5 \mathrm{mV}$ every 10 seconds. Current was recorded every 6 seconds.

All electrophysiologic measurements were performed on duplicate or triplicate filters; statistics were analyzed using Instat (GraphPad Software for Science Inc., San Diego, California, USA), and data were graphed using either SigmaPlot or DeltaGraph.

Mannitol flux. Determination of $\left[{ }^{3} \mathrm{H}\right]$-mannitol flux was carried out as described by McCarthy et al. (22) on monolayers of uninduced or induced cells. Briefly, cells were plated on Transwell filters (Corning Inc. Life Sciences) in the presence or absence of doxycycline. After 4 days, media was replaced with fresh media (with or without doxycycline) supplemented with $1 \mathrm{mM}$ mannitol in both the apical and basal chambers. In addition, the apical media $(0.5 \mathrm{ml})$ contained $4 \mu \mathrm{Ci}(1$ $\mu \mathrm{Ci} / \mu \mathrm{M}) \quad\left[{ }^{3} \mathrm{H}\right]$-mannitol (Amersham Pharmacia Biotech Inc.) at tracer levels for measurement of apical to basolateral diffusion. At 0, 30, 60, 120, and $180 \mathrm{~min}$ utes, $100 \mu \mathrm{l}$ of media was removed from the basal compartment and replaced with $100 \mu \mathrm{l}$ of fresh media with $1 \mathrm{mM}$ mannitol, with or without doxycycline. The 100$\mu \mathrm{l}$ samples were added to $5 \mathrm{ml}$ Opti-Fluor (Packard Instrument Co., Meriden, Connecticut, USA) and radioactivity determined in a Wallac 1409 liquid-scintillation counter. Values were corrected for dilution of label due to sample removal.

Transcellular transport-inhibitor studies. Base-line measurements were taken in buffer A on identically treated filters containing uninduced or induced MDCK cell
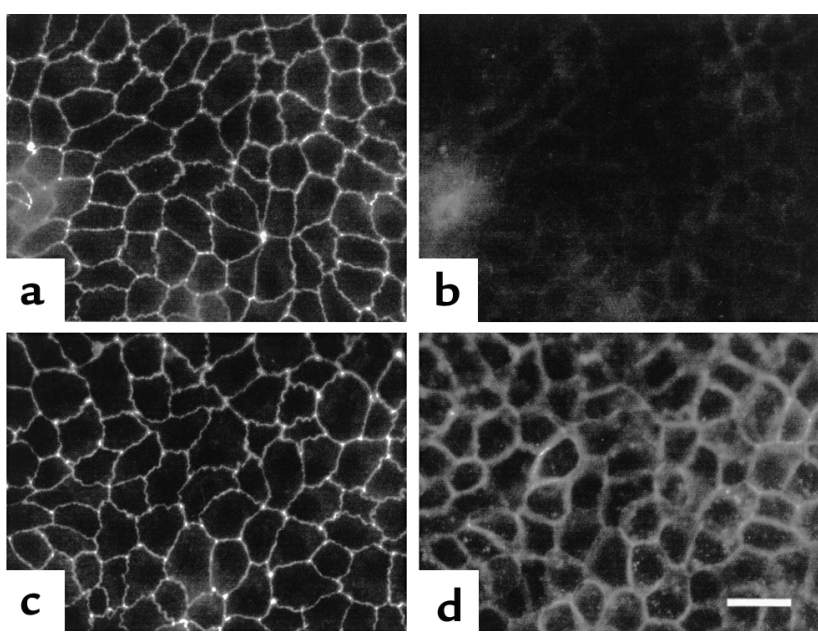

\section{Figure 2}

Immunofluorescent colocalization of ZO-1 and claudin-4 in tet-off claudin-4-transfected MDCK cells. Cells were plated on filters and cultured for 4 days in the presence ( $\mathbf{a}$ and $\mathbf{b}$ ) or absence ( $\mathbf{c}$ and $\mathbf{d}$ ) of $50 \mathrm{ng} / \mathrm{ml}$ doxycycline and stained for ZO-1 (a and $\mathbf{c}$ ) and claudin-4 (b and $\mathbf{d}$ ). ZO-1 is narrowly focused at the apical junction, while claudin-4 in both uninduced (b) and induced (d) MDCK cells is concentrated at cell-cell borders. 
monolayers. Buffer A was aspirated, and fresh buffer A containing the indicated drug concentrations was used to wash monolayers once and then added to the apical and basal chamber compartments. Conductance and transmonolayer potential were measured, and the apical buffer A was replaced (after one wash) with buffer B containing the indicated drug. Transepithelial electrical potential and conductance were determined as described previously. The effects of addition of drugs to empty filters were also determined. Drugs used were amiloride $(0.1 \mathrm{mM})$, bumetanide $(0.01 \mathrm{mM})$, and 4-acetamido-4'isothiocyanato-stilbene-2, 2'-disulfonic acid (SITS; 0.1 mM) (Sigma Chemical Co., St. Louis, Missouri, USA).

\section{Results}

Induced expression of claudin-4 does not alter the levels of other tight-junction proteins. Claudin-4 is normally detectable in MDCK cells (23). Thus, we would ideally have introduced an epitope-tagged version of claudin- 4 so that its expression and cellular location could be distinguished from endogenous protein. However, we chose not to
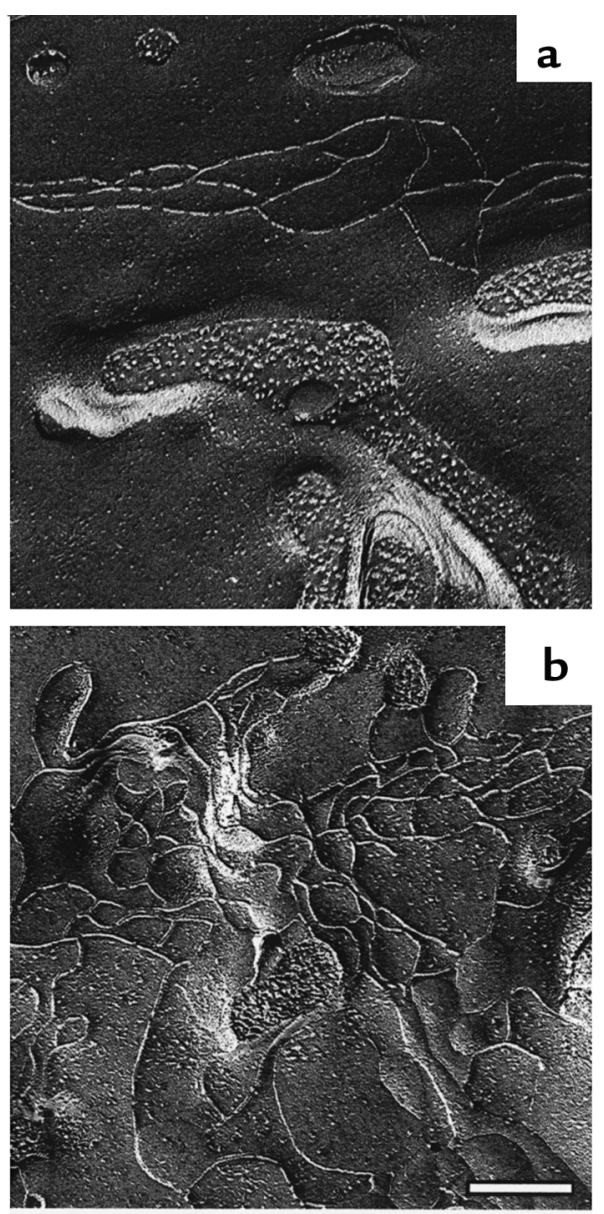

\section{Figure 3}

Freeze-fracture analysis of claudin-4-overexpressing MDCK cells. Uninduced MDCK cells show the normal parallel tightjunction strands with angled cross bridges (a). Induced MDCK cells show a pattern of fibrils that is less parallel, deeper, and more complex (b). $\times 38,000$. Bar, $200 \mathrm{~nm}$. use tagged constructs for specific reasons. First, we observed (data not shown) that tagged proteins were either not expressed or remained in an intracellular compartment when a VSV-G tag sequence was placed at any of three different positions in the sequence. Second, previously published results demonstrated functional differences between carboxy-terminal tagged and untagged claudin-1 when expressed in MDCK cells (13). Consequently, we used untagged wild-type human claudin-4 in an inducible expression system, and all comparisons were based on the difference within individual clones between their uninduced and induced states. This also eliminated the potential and significant problem of clonal variation. Tet-off MDCK cells were transfected with pTRE-(claudin-4), and selection of transfected clones was performed by immunoblot analysis of hygromycin-resistant colonies. Analysis of 40 hygromycin-resistant colonies provided 15 clones showing strong induction of a protein of the expected size, about $22 \mathrm{kDa}$. Five clones were chosen for further study because they had low basal levels of claudin-4 on immunoblots and were highly inducible.

Figure 1 shows the induction of claudin- 4 after 4 days in three representative clones. Variable levels of basal expression are seen in each. A strong induction of between fourfold and tenfold was reproducibly seen in all clones at 4 days. No change in the levels of claudin- $1 / 3$ and claudin- 2 were observed coincident with induction of higher levels of claudin-4 (Figure 1, b and c). In addition, induction of claudin- 4 has no obvious effect on the levels of another transmembrane protein, occludin (Figure $1 \mathrm{~d}$ ), or a cytoplasmic protein that binds to claudins, ZO-1 (Figure 1e). These results are similar to the lack of effect on ZO-1 and occludin associated with overexpressing claudin-1 in MDCK cells (13). We conclude that any physiologic changes associated with the overexpression of claudin-4 may be primary and not secondary to changes in the levels of other junction proteins.

Immunolocalization of claudin-4 and freeze-fracture electron microscopy. We next used indirect immunofluorescence to determine the cellular localization of overexpressed claudin-4 (Figure 2). A significant increase in staining at cell borders was reproducibly observed in all clones (Figure 2, b and d). This localization was also seen in uninduced cells and was described previously by Sonoda et al. in untransfected MDCK cells (23). In contrast, both of the tight-junction proteins ZO-1 (Figure 2, a and c) and occludin (not shown) were concentrated at the apical junction contacts. However, existence of minor lateral pools of ZO-1 and occludin could not be ruled out using these techniques, and, in fact, McCarthy et al. (13) demonstrated rare inclusion of occludin within lateral claudin-1 fibrils.

We performed freeze-fracture electron microscopy to determine whether overexpression resulted in changes in junction strands. Two separate clonal lines for both uninduced (21 total replicas) and induced cells (20 total replicas) were studied and showed a highly consistent difference. Uninduced MDCK cells (Figure 3a) 


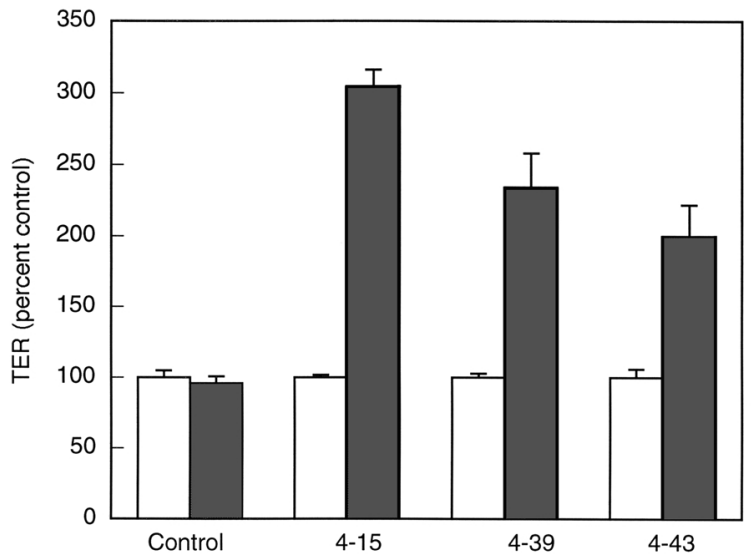

Figure 4

Claudin- 4 overexpression results in increased TER. MDCK cell clones described in Figure 2 were plated onto Snapwell inserts and cultured in the presence (open bars) or absence (filled bars) of $50 \mathrm{ng} / \mathrm{ml}$ doxycycline for 4 days. TER was determined as described in Methods; data represent the mean and SEM of determinations from triplicate Snapwells. TER in claudin-4-overexpressing MDCK cells, but not in parental tet-off cells, in the absence of doxycycline is significantly higher $(P<0.005)$ than in doxycycline-treated cells. Base-line TERs averaged about $40 \Omega \mathrm{cm}^{2}$; after claudin- 4 induction, TER was between 100 and $200 \Omega \mathrm{cm}^{2}$.

showed a freeze-fracture pattern typical of MDCK cells (24), with a few parallel fibrils and angled cross bridges oriented along the apical-lateral border. In contrast, in the cells overexpressing claudin- 4 the total content of strands was increased, and the pattern was more elaborate (Figure $3 b$ ). In addition to the parallel apical strand pattern, numerous additional strands loop down and back up on the lateral surface of the cell. The laterally displaced strands also formed cell-cell contacts as determined by their continuity at P-to-E face transitions (Figure $3 \mathrm{~b}$ ). The increase in strands was found around the entire tight-junction belt of the induced MDCK cells. It seems likely that at least some of the overexpressed claudin- 4 is incorporated into these new tight-junction fibrils, and we next determined whether this might induce changes in paracellular permeability.

Induction of claudin-4 increases TER. Induction of claudin- 4 for 4 days led to an increase in TER by approximately twofold to threefold in five separate clonal lines (three lines shown in Figure 4). Each clone appeared to reach a reproducible maximal increase by 4 days that was approximately proportional to the overall increase in claudin-4 (compare with immunoblots in Figure 1a). A control clone transfected with empty vector showed no change in TER in response to removing doxycycline. In monolayers of low resistance, such as these, the principal route for electrical conductance is paracellular. Thus, the increase can be tentatively interpreted as resulting from an increase in the paracellular resistance $(1,25)$. Others have reported a similar increase in TER in response to overexpression of claudin-1 in MDCK cells $(12,13)$.
Regulated expression of claudin-4 increases TER through a selective decrease in $P_{\mathrm{Na}^{+}}$without a significant effect on $P_{\mathrm{Cl}}$. The increase in TER, i.e., decrease in paracellular conductance, associated with overexpression of claudin- 4 could result from either a nonselective simultaneous decrease in the permeability for both cations and anions or differential effects on one or both. The latter outcome would imply that claudin-4 confers the ability to discriminate between $\mathrm{Na}^{+}$and $\mathrm{Cl}^{-}$. To discriminate between these possibilities we measured the transepithelial voltage resulting from imposition of a transmonolayer gradient in $\mathrm{NaCl}$ concentration (Table 1).

In five separate clones, overexpression of claudin-4 resulted in a highly reproducible and consistent increase in the ratio of Cl:Na permeability and simultaneous decrease in overall conductance, both approximately twofold (Table 1). These values were used to calculate the individual permeabilities for $\mathrm{Na}^{+}$and $\mathrm{Cl}^{-}$ ions (21). While $P_{\mathrm{Na}}{ }^{+}$was consistently decreased by $50 \%$ in five separate clones, there was no statistically significant effect on $P_{\mathrm{Cl}^{-}}$. The absolute value of the observed dilution potential was independent of the direction of the applied $\mathrm{NaCl}$ gradient (data not shown), consistent with the effect resulting from a change in ion discrimination in the paracellular and not the transcellular pathway. In addition, control treatment of the parental cell lines with doxycycline resulted in no changes in dilution potentials (data not shown). Finally, bi-ionic potential experiments demonstrated no change in the rank order and relative values of permeability for the other alkali metal cations $\left(\mathrm{K}^{+}>\right.$ $\left.\mathrm{Na}^{+}>\mathrm{Li}^{+}=\mathrm{Rb}^{+}>\mathrm{Cs}^{+}\right)$in claudin-4-overexpressing cells $(1.0>0.88>0.65-0.68>0.32)$ compared with uninduced cells $(1.0>0.8>0.58-0.60>0.22)$. Very similar values were reported for MDCK cells previously (26). We conclude that overexpression of claudin-4 results in a decrease in transmonolayer electrical conductance that is attributable to a selective discrimination against the paracellular passage of $\mathrm{Na}^{+}$without a significant influence on the permeability of $\mathrm{Cl}^{-}$. In addition, the permeability for all the alkali metal cations is decreased, as implied by the lack of effect of claudin-4 on their permeabilities relative to $\mathrm{Na}^{+}$.

\section{Table 1}

Overexpression of claudin- 4 alters transepithelial $\mathrm{Na}^{+}$conductance across MDCK cell monolayers

$\begin{array}{lcc} & \text { Uninduced cells (+ dox) } & \text { Induced cells (- dox) } \\ \Delta V_{T}(\mathrm{mV})^{\mathrm{A}} & 7.00 \pm 0.51 & 4.15 \pm 0.63^{\mathrm{E}} \\ G_{T}\left(\mathrm{mS} / \mathrm{cm}^{2}\right)^{\mathrm{B}} & 26.37 \pm 1.43 & 12.64 \pm 1.99^{\mathrm{E}} \\ P_{\mathrm{Cl}} / P_{\mathrm{Na}} \mathrm{C} & 0.396 \pm 0.033 & 0.601 \pm 0.050^{\mathrm{F}} \\ P_{\mathrm{Na}}\left(10^{-6} \mathrm{~cm} / \mathrm{s}\right)^{\mathrm{D}} & 31.09 \pm 2.23 & 17.63 \pm 2.32^{\mathrm{G}} \\ P_{\mathrm{Cl}}\left(10^{-6} \mathrm{~cm} / \mathrm{s}\right)^{\mathrm{D}} & 12.01 \pm 0.95 & 9.76 \pm 0.94^{\mathrm{H}}\end{array}$

Mean \pm SEM of five separate claudin-4-overexpressing MDCK cell clones; each measurement made on duplicate wells. ${ }^{\mathrm{A}}$ Dilution potential. ${ }^{\mathrm{B}} \mathrm{G}_{T} .{ }^{\mathrm{C}} \mathrm{C}$ alculated using the constant field equation (20). ${ }^{D}$ Calculated using the Kimizuka-Koketzu equation (21). ${ }^{\mathrm{E}} P<0.005 .{ }^{\mathrm{F}} P=0.0063 .{ }^{\mathrm{G}} P=0.0102 .{ }^{\mathrm{H}} \mathrm{Not}$ significant by Student's $t$ test compared with uninduced cells. 

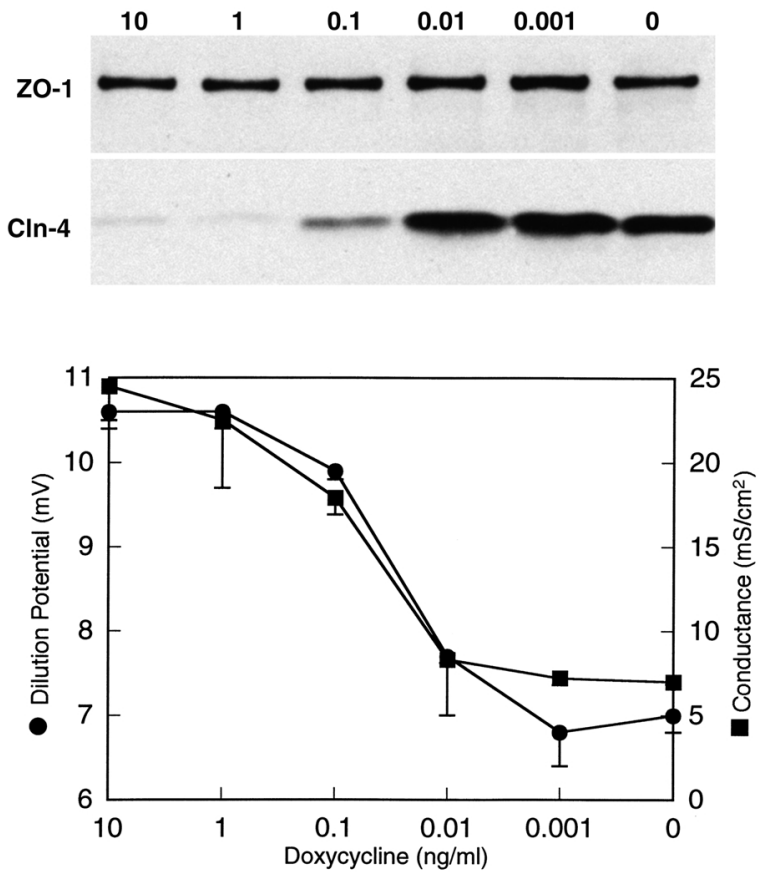

Figure 5

Decreases in the dilution potential and conductance, in response to doxycycline treatment, correlate with increases in claudin-4 expression. Top panel, immunoblot of cells plated on Snapwells and treated with varying doses of doxycycline $(10,1,0.1,0.01,0.001$, and 0 $\mathrm{ng} / \mathrm{ml}$ ) for 4 days. Snapwells were removed and used for electrophysiologic measurements (lower panel); after measurement, cells on filters were lysed, proteins separated by SDS-PAGE, and immunoblotted for claudin-4 and ZO-1. Dilution potential (filled circles) and conductance (filled squares) were determined as described in the Methods section.

To implicate claudin- 4 more directly as the cause of the altered paracellular permeability we attempted to correlate conductance and dilution potential with a range of protein expression levels by varying the doxycycline levels. A representative dose-response relationship is presented in Figure 5. To induce a graded increase in claudin- 4 expression, cells were plated onto Snapwell filters and immediately placed in media containing $10,1,0.1,0.01,0.001$, and $0 \mathrm{ng} / \mathrm{ml}$ doxycycline and were cultured for 4 days. Triplicate filters were used for measurements of conductance and dilution potential, after which filter inserts were excised and extracted for immunoblot analysis. The kinetics and magnitude of change in conductance and dilution potential were indistinguishable and correlated directly with the increase in expression of claudin-4. By comparison the level of ZO-1 was unchanged (Figure 5). This direct dose-response correlation is consistent with the conclusion that claudin- 4 is directly responsible for the decrease in paracellular conductance by selectively decreasing the permeability for $\mathrm{Na}^{+}$ions.

Two additional approaches were used to further verify that the increase in TER resulted from an increase in paracellular rather than transcellular resistance.
First, the current-voltage curves were observed to be linear across a wide voltage range in both uninduced and induced cells $(-30$ to $+30 \mathrm{mV})$. The slope of the line was increased, however, in induced cells, revealing the increased electrical resistance. This behavior is consistent with conductance through the relatively large and less-selective aqueous spaces of the paracellular pathway rather than through the limited capacity and high selectivity of transmembrane carriers. As a control, doxycycline itself had no effect on the slopes observed in untransfected cells. Second, inhibitors of three major transcellular pathways were added to uninduced and induced cells to see if they altered the conductance or dilution potential. These included amiloride, bumetanide, and SITS to block $\mathrm{Na}^{+}$channels, NKCCcotransporters and anion exchangers, respectively (27). The dilution potentials were not significantly altered by these drugs in either uninduced or induced cells (data not shown). These results are also consistent with idea that the change in $\mathrm{Na}^{+}$permeability in claudin4-overexpressing MDCK cells is not due to changes in the transcellular channels.

Mannitol flux is unaffected by overexpressing claudin-4. A decrease in the dimensions of the paracellular pathway might also contribute to the decrease in conductance resulting from overexpression of claudin- 4 . To test this possibility we compared the unidirectional diffusion of $\left[{ }^{3} \mathrm{H}\right]$-mannitol across uninduced and induced cell monolayers. Four clones were plated in triplicate in the presence or absence of doxycycline. After 4 days, the flux of $\left[{ }^{3} \mathrm{H}\right]$-mannitol was observed to be unaffected by induction of claudin-4 (Figure 6). Flux is influenced by the area available for diffusion through the tight junction as well as the length and width of the lateral inter-

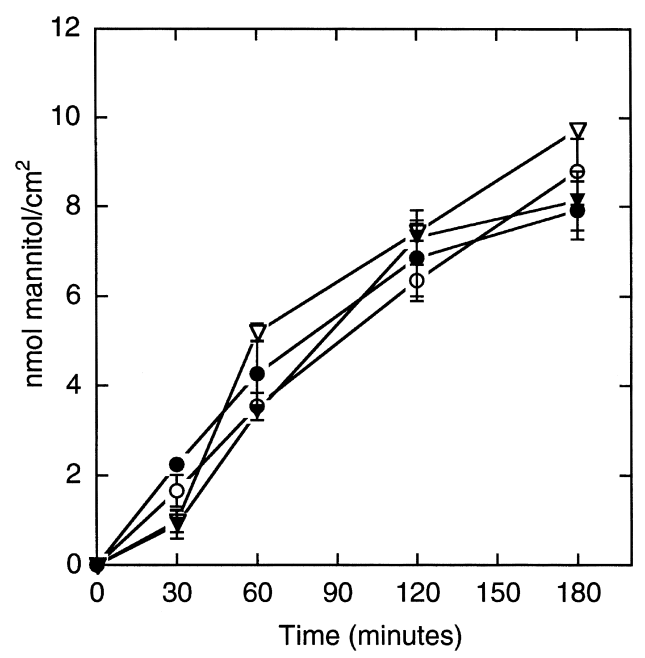

Figure 6

Induction of claudin-4 in MDCK cells does not alter transepithelial $\left[{ }^{3} \mathrm{H}\right]$ mannitol flux. Cells were plated as described in the legend for Figure 1 and cultured for 4 days in the presence (open symbols) or absence (closed symbols) of $50 \mathrm{ng} / \mathrm{ml}$ doxycycline. Circles indicate clone 15, and triangles represent clone 39 . There were no differences in mannitol flux either between clones or with or without claudin-4 induction. 
cellular space (28); the flux results suggest these dimensions are not changed by overexpressing claudin- 4 .

\section{Discussion}

In this study we begin to address the question of whether members of the claudin family confer ionic selectivity to the paracellular pathway. We observed that overexpression of claudin-4 in MDCK cells decreases transmonolayer conductance by decreasing paracellular $\mathrm{Na}^{+}$permeability. There is no effect on the permeability for $\mathrm{Cl}^{-}$ or flux for a noncharged solute. The close correlation between the level of claudin- 4 expression and change in both conductance and ionic selectivity suggest it is directly responsible for these changes. These results are consistent with a model in which claudin- 4 forms channels through the tight junction that discriminate against $\mathrm{Na}^{+}$ions and are indifferent to $\mathrm{Cl}^{-}$ions. The results of biionic potential studies imply that the permeability was also decreased proportionately for the other alkali metal cations, $\mathrm{K}^{+}, \mathrm{Li}^{+}, \mathrm{Rb}^{+}$, and $\mathrm{Cs}^{+}$. Although recent studies by Inai et al. (12) and McCarthy et al. (13) showed that overexpression of claudin- 1 increased TER in MDCK cells, the present data are the first direct evidence, to our knowledge, that overexpression of a claudin can confer paracellular ionic discrimination. Previous studies have documented the varied distribution of individual claudins $(5,14)$ and led to the speculation they might be responsible for the variable electrical properties of tight junctions in different tissues. Demonstration of the ability of claudin-4 to alter $\mathrm{Na}^{+}$selectivity supports this model and is an important first step in understanding underlying mechanisms responsible for tissue-specific tight-junction properties.

Several lines of evidence strongly suggest claudin- 4 is affecting the paracellular pathway. First, paracellular conductance greatly exceeds transcellular conductance in MDCK cells, therefore if claudin- 4 increases TER it must do this through an effect on tight junctions (20). Second, the number of junction strands is increased by overexpression of claudin-4, while the levels of several other strand proteins are unaffected. Thus we presume the additional strands are made predominately of claudin- 4 in a position to influence the barrier characteristics of the junction. This does not rule out the possibility that claudin on the lateral cell surface, not assembled into strands, can influence paracellular permeability, and this could be tested experimentally by using osmotic gradients to shrink or swell the lateral intercellular space. Third, the instantaneous current-voltage relationships were linear and symmetrical in both uninduced and induced MDCK cells. Transmembrane carriers would be expected to have a limited capacity to conduct current and result in a nonlinear I-V relationship. The observed lack of saturation is consistent with the major avenue of conductance being between the cells rather than through membrane carriers. Finally, amiloride, bumetanide, and SITS were used to block major transcellular ion-transporting channels. Since dilution potentials in both uninduced and induced cells were unaffected by the presence of these inhibitors, it is likely that the potentials were generated by the paracellular rather than transcellular movement of $\mathrm{Na}^{+}$and $\mathrm{Cl}^{-}$ions.

It should be noted that we chose to ignore any contribution to the dilution potential from ions other than $\mathrm{Na}^{+}$and $\mathrm{Cl}^{-}$. This is commonly done and valid to a first approximation since the other ions are present at lower concentrations and their transmonolayer ratios were kept constant during imposition of the $\mathrm{NaCl}$ gradient. It is possible that the other ions compete with $\mathrm{Na}^{+}$and $\mathrm{Cl}^{-}$differently at the two $\mathrm{NaCl}$ concentrations. This anomalous behavior would be expected to contribute equally to the dilution potential in both uninduced and induced cells. Thus, the increase in $P_{\mathrm{Cl}} / P_{\mathrm{Na}}$ observed with overexpression of claudin- 4 must reveal a true change in the relative selectivity even if the exact magnitudes are approximations.

Overexpressing claudin-4 does not stimulate an overall increase in the levels of other tight-junction components, consistent with a previous study by McCarthy et al. on claudin-1 (13). Notably, ZO-1 levels do not change in the face of claudin- 4 induction. One postulated role for the $\mathrm{ZO}$ proteins is to scaffold the integral membrane proteins and link them to the actin cytoskeleton $(17,29$, 30). The physiologic effects of a large increase in claudin-4 without an increase in ZO-1 suggest that either this linkage is not required for barrier function or that the $\mathrm{ZO}$ proteins or other components involved in the linkage are not limiting. That levels of occludin and the other claudins measured do not diminish in the face of claudin- 4 overexpression suggests that claudin- 4 does not act to replace these integral membrane tightjunction proteins, but adds to them. Since the composition of the tight junction particle is unknown, it is impossible to predict how overexpression of claudin- 4 alters the stoichiometry of claudins and occludin in the new tight-junction strands.

Many studies have now shown dissociation between changes in the barrier properties of electrical conductance for ions and the flux for uncharged solutes (2, 31). Transmonolayer conductance is an instantaneous measure of ion permeability across the paracellular pathway during imposition of an electrical field, reflecting only the number and nature of channels at that instant. This property varies over five orders of magnitude among normal tissues, yet their size selectivity for noncharged solutes is very similar (1). This supports the idea that the channels discriminate on the basis of charge but are of the same size. Mannitol is a small, uncharged solute commonly used to probe the size of the paracellular channels; the flux for mannitol is normally measured over minutes to hours. Although conductance and flux often change together when tight-junction function is compromised, there is considerable support for the idea that the barriers measured by these two methods are functionally and physically distinct. The longer times over which flux is measured implies that if strands were labile, i.e., separated and reformed over time, then the paracellular 
route available for diffusion would be different from that measured by electrical conductance and not dependent on claudin-based channels. A number of recent studies document increased TER (or decreased conductance) with either no effect on or seemingly paradoxical increases in mannitol flux. Examples include EGF treatment on LLC-PK 1 cells (27), overexpression of occludin $(31,32)$, overexpression of claudin-1 (13), and RhoA activation in MDCK cells (33). The observation that overexpressing claudin-4 decreases ionic conductance without altering the rate of mannitol flux is consistent with the idea that the selectivity but not the size of the paracellular channels has been altered. Although a number of models have been proposed, reasons for the lack of correlation between the paracellular movement of ions, water, and small nonionic solutes like mannitol are still unclear (2).

The ion selectivity of MDCK tight junctions was first determined nearly 25 years ago $(20,26,34)$. In those studies, MDCK cells were found to be cation selective and the rank order of cation permeability was determined, but there were some differences among the studies. Our results in uninduced tet-off MDCK cells corresponded well with those published by Rabito et al. (26), although our cells were slightly less cation selective $\left(P_{\mathrm{Na}} / P_{\mathrm{Cl}}=2.5\right.$ in our cells compared with $P_{\mathrm{Na}} / P_{\mathrm{Cl}}=$ 4.4). Induction of claudin- 4 resulted in a specific decrease in absolute sodium permeability and changed the $P_{\mathrm{Na}} / P_{\mathrm{Cl}}$ to 1.6. Although our study is the first report in which a change in ionic selectivity can be attributed to overexpression of a specific tight-junction protein, there are previous reports of experimental treatments that alter the ionic selectivity of the tight junction. These include the effects of EGF on LLC-PK 1 cells (27) and TNF- $\alpha$ (35) on CACO- 2 cells, among others. One possible explanation is that these treatments alter claudin expression profiles in the tight junction, with concomitant changes in paracellular permeability characteristics. This form of regulation of paracellular permeability would then also be expected to exist in vivo.

The molecular basis by which claudin- 4 influences paracellular selectivity remains unknown. A likely possibility is that claudins form selective channels in the tight junction through their oligomerization into particles and lateral cell-cell contacts of the particles. The variable amino acid sequences of their extracellular domains, particularly charged side chains, might be positioned to modify the selectivity of the channels (35). Consistent with this model is the observation that the extracellular sequences of claudin-16/paracellin are among the most acidic. Indirect evidence suggests claudin-16 permits the paracellular passage of $\mathrm{Mg}^{++}$and other cations (6). In preliminary studies, we observe that overexpressing claudin-2 in MDCK cells results in an increase in paracellular conductance resulting from an increase in $P_{\mathrm{Na}+}$. This behavior is opposite that associated with overexpression of claudin-4, again supporting the idea that each claudin contributes a different channel-like property. Since it has been demonstrated that some combi- nations of various claudins can interact both within strands and across cells (36-38), the combinatorial possibilities offered by all 20 claudins could easily provide extensive physiologic variability among tissues. Until we better understand the nature of the tight-junction particle and how it combines with partners on the opposing cell as well as with plaque proteins, our interpretation of the electrophysiologic data will be necessarily limited.

\section{Acknowledgments}

These studies were supported by grants from the NIH (DK-45134, DK-34989, and DK-38979). We are also indebted to Alan S. Fanning, Laura Mitic, Emile Boulpaep, John Geibel, and Lukas Landmann for invaluable discussion or technical assistance.

1. Powell, D.W. 1981. Barrier function of epithelia. Am. J. Physiol.
241:G275-G288.

2. Madara, J.L. 1998. Regulation of the movement of solutes across tight junctions. Annu. Rev. Physiol. 60:143-159.

3. Reuss, L. 1991. Tight junction permeability to ions and water. In Tight junctions. M. Cereijido, editor. CRC Press Inc. Boca Raton, Florida, USA. 49-66.

4. Furuse, M., Fujita, K., Hiiragi, T., Fujimoto, K., and Tsukita, S. 1998. Claudin-1 and -2: novel integral membrane proteins localizing at tight junctions with no sequence similarity to occludin. J. Cell. Biol. 141:1539-1550.

5. Morita, K., Furuse, M., Fujimoto, K., and Tsukita, S. 1999. Claudin multigene family encoding four-transmembrane domain protein components of tight junction strands. Proc. Natl. Acad. Sci. USA. 96:511-516.

6. Simon, D.B., et al. 1999. Paracellin-1, a renal tight junction protein required for paracellular $\mathrm{Mg} 2+$ resorption. Science. 285:103-106.

7. Gow, A., et al. 1999. CNS myelin and sertoli cell tight junction strands are absent in Osp/claudin-11 null mice. Cell. 99:649-659.

8. Mitic, L.L., Van Itallie, C.M., and Anderson, J.M. 2000. Molecular physiology and pathophysiology of tight junctions. I. Tight junction structure and function: lessons from mutant animals and proteins. Am. J. Physiol. 279:G250-G254.

9. Itoh, M., et al. 1999. Direct binding of three tight junction-associated MAGUKs, ZO-1, ZO-2, and ZO-3 with the COOH termini of claudins. J. Cell. Biol. 147:1351-1363.

10. Furuse, M., Sasaki, H., Fujimoto, K., and Tsukita, S. 1998. A single gene product, claudin-1 or -2 , reconstitutes tight junction strands and recruits occludin in fibroblasts. J. Cell. Biol. 143:391-401.

11. Wilcox, E.R., et al. 2001. Mutations in the gene encoding tight junction claudin-14 cause autosomal recessive deafness DFNB29. Cell. 104:165-172.

12. Inai, T., Kobayashi, J., and Shibata, Y. 1999. Claudin-1 contributes to the epithelial barrier function in MDCK cells. Eur. J. Cell. Biol. 78:849-855.

13. McCarthy, K.M., et al. 2000. Inducible expression of claudin-1-myc but not occludin-VSV-G results in aberrant tight junction strand formation in MDCK cells. J. Cell. Sci. 113:3387-3398.

14. Rahner, C., Mitic, L.L., and Anderson, J.M. 2001. Heterogeneity in expression and subcellular localization of claudin-2, 3, 4 and 5 in the rat liver, pancreas, and gut. Gastroenterology. 120:411-422.

15. Morita, K., Sasaki, H., Furuse, M., and Tsukita, S. 1999. Endothelial claudin: claudin-5/TMVCF constitutes tight junction strands in endothelial cells. J. Cell. Biol. 147:185-194.

16. Morita, K., Sasaki, H., Fujimoto, K., Furuse, M., and Tsukita, S. 1999. Claudin-11/OSP-based tight junctions of myelin sheaths in brain and Sertoli cells in testis. J. Cell. Biol. 145:579-588.

17. Fanning, A.S., Jameson, B., Jesaitis, L.A., and Anderson, J.M. 1998. The tight junction protein $\mathrm{ZO}-1$ establishes a link between the transmembrane protein occludin and the actin cytoskeleton. J. Biol. Chem. 273:29745-29753.

18. Medina, R., Anderson, J.M., and Van Itallie, C.M. 2000. Occludin localization at the tight junction requires the second extracellular loop. $J$. Membr. Biol. 178:235-247.

19. Gorodeski, G.I., Peterson, D.E., DeSantis, B.J., and Hopfer, U. 1996. Nucleotide receptor-mediated decrease of tight-junctional permeability in cultured human cervical epithelium. Am. J. Physiol. 270:C1715-C1725.

20. Cereijido, M., Robbins, E.S., Dolan, W.J., Rotunno, C.A., and Sabatini, D.D. 1978. Polarized monolayers formed by epithelial cells on a permeable and translucent support. J. Cell. Biol. 77:853-880.

21. Delamere, N.A., and Duncan, G. 1977. A comparison of ion concentrations, potentials and conductances of amphibian, bovine and cephalod lenses. J. Physiol. 272:167-186.

22. McCarthy, K.M., et al. 1996. Occludin is a functional component of the 
tight junction. J. Cell. Sci. 109:2287-2298.

23. Sonoda, N., et al. 1999. Clostridium perfringens enterotoxin fragment removes specific claudins from tight junction strands. Evidence for direct involvement of claudins in tight junction barrier. J. Cell. Biol. 147:195-204.

24. Stevenson, B.R., Anderson, J.M., Goodenough, D.A., and Mooseker, M.S 1988. Tight junction structure and ZO-1 content are identical in two strains of Madin-Darby canine kidney cells which differ in transepithelial resistance. J. Cell. Biol. 107:2401-2408.

25. Diamond, J. 1977. The epithelial junction: bridge, gate and fence. Physiologist. 20:10-18.

26. Rabito, C.A., Tchao, R., Valentich, J., and Leighton, J. 1978. Distribution and characteristics of the occluding junctions in a monolayer of a cell line (MDCK) derived from canine kidney. J. Membr. Biol. 43:351-365.

27. Saladik, D.T., Soler, A.P., Lewis, S.A., and Mullin, J.M. 1995. Cell division does not increase transepithelial permeability of $\mathrm{LLC}_{\mathrm{L}} \mathrm{PK}_{1}$ cell sheets. Exp. Cell. Res. 220:446-455.

28. Van Itallie, C., and Anderson, J.M. 2000. Molecular structure and regulation of tight junctions. In Current topics in membranes. K.E. Barrett and M. Donowitz, editors. Academic Press. San Diego, California, USA. 163-186.

29. Wittchen, E.S., Haskins, J., and Stevenson, B.R. 1999. Protein interactions at the tight junction. Actin has multiple binding partners, and $\mathrm{ZO}$ 1 forms independent complexes with ZO-2 and ZO-3. J. Biol. Chem. 274:35179-35185.

30. Itoh, M., Nagafuchi, A., Moroi, S., and Tsukita, S. 1997. Involvement of
ZO-1 in cadherin-based adhesion through its direct binding to $\alpha$ catenin and actin filaments. J. Cell. Biol. 138:181-192.

31. Balda, M.S., et al. 1996. Functional dissociation of paracellular permeability and transepithelial electrical resistance and disruption of the apical-basolateral intramembrane diffusion barrier by expression of a mutant tight junction membrane protein. J. Cell. Biol. 134:1031-1049.

32. McClane, B.A. 1994. Clostridium perfringens enterotoxin acts by producing small molecule permeability alterations in plasma membranes. Toxicology. 87:43-67.

33. Hasegawa, H., et al. 1999. Opposite regulation of transepithelial electrical resistance and paracellular permeability by Rho in Madin-Darby canine kidney cells. J. Biol. Chem. 274:20982-20988.

34. Misfeldt, D.S., Hamamoto, S.T., and Pietelka, D.R. 1976. Transepithelial transport in cell culture. Proc. Natl. Acad. Sci. USA. 73:1212-1216.

35. Marano, C.W., Lewis, S.A., Garulacan, L.A., Soler, A.P., and Mullin, J.M. 1998. Tumor necrosis factor-alpha increases sodium and chloride conductance across the tight junction of CACO-2 BBE, a human intestinal epithelial cell line. J. Membr. Biol. 161:263-274.

36. Wong, V., and Goodenough, D.A. 1999. Paracellular channels! Science. 285:62

37. Furuse, M., Sasaki, H., and Tsukita, S. 1999. Manner of interaction of heterogeneous claudin species within and between tight junction strands. J. Cell. Biol. 147:891-903.

38. Kubota, K., et al. 1999. Ca(2+)-independent cell-adhesion activity of claudins, a family of integral membrane proteins localized at tight junctions. Curr. Biol. 9:1035-1038. 